\title{
Universality class and exact phase boundary in the superradiant phase transition
}

\author{
Wei-Feng Zhuang, ${ }^{1}$ Bin Geng, ${ }^{1}$ Hong-Gang Luo, ${ }^{2,3}$ Guang-Can Guo, ${ }^{1,4,5}$ and Ming Gong ${ }^{1,4,5, *}$ \\ ${ }^{1}$ CAS Key Laboratory of Quantum Information, University of Science \\ and Technology of China, Hefei, 230026, Peoples Republic of China \\ ${ }^{2}$ School of Physical Science and Technology, Lanzhou 730000, China \\ ${ }^{3}$ Beijing Computational Science Research Center, Beijing 100084, China \\ ${ }^{4}$ Synergetic Innovation Center of Quantum Information and Quantum Physics, \\ University of Science and Technology of China, Hefei, Anhui 230026, China \\ ${ }^{5}$ CAS Center For Excellence in Quantum Information and Quantum Physics, \\ University of Science and Technology of China, Hefei, Anhui 230026, China
}

(Dated: August 10, 2020)

\begin{abstract}
The Dicke model and Rabi model can undergo phase transitions from the normal phase to the superradiant phase at the same boundary, which can be accurately determined using some approximated approaches. The underlying mechanism for this coincidence is still unclear and the universality class of these two models is elusive. Here we prove this phase transition exactly using the path-integral approach based on the faithful Schwinger fermion representation, and give a unified phase boundary condition for these models. We demonstrate that at the phase boundary, the fluctuation of the bosonic field is vanished, thus it can be treated as a classical field, based on which a much simplified method to determine the phase boundary is developed. This explains why the approximated theories by treating the operators as classical variables can yield the exact boundary. We use this method to study several similar spin and boson models, showing its much wider applicability than the previously used approaches. Our results demonstrate that these phase transitions belong to the same universality by the classical Landau theory of phase transitions, which can be confirmed using the platforms in the recent experiments.
\end{abstract}

The Dicke model has been studied for more than half a century [1-4]. This model considers the coupling between $N$ identical atoms (or two-level systems) with a bosonic field, which can be written as

$$
\mathcal{H}=\omega b^{\dagger} b+\sum_{i=1}^{N} \frac{\Omega}{2} \sigma_{i}^{z}+\frac{g}{\sqrt{N}} \sigma_{i}^{x}\left(b+b^{\dagger}\right) .
$$

Here $b$ is the annihilate operator for the bosonic field, $\sigma_{i}^{x}, \sigma_{i}^{z}$ are the Pauli operators for the $i$-th atom and $N$ is the total number of atoms. This model undergoes a phase transition from a normal phase to a superradiant phase at $g_{c}^{2}=\frac{\Omega \omega}{4} \operatorname{coth} \frac{\beta \Omega}{2}[5-7]$, where $\beta=1 / k_{B} T$, with $k_{B}$ is the Boltzmann constant and $T$ is the temperature. The phase transition can be obtained from the Holstein-Primakoff (HP) approximation [8,9] and semiclassical approximation $[10,11]$, in which negative or complex eigenvalues mark the ground state instability. It is challenging to be realized with atoms in radiation-plusmatter field due to not only the required large density, but also the no-go theorem [12-15]. However, it can be realized with ultracold atoms [16-22], driven-dissipative quantum simulators $[23,24]$ and spin-orbit coupled condensates in a trap [25] and electron gases in a cavity [26].

Recently, the phase transition with only one atom has attracted widespread attention [27-30]. When $N=1$, Eq. 1 is reduced to the exact solvable quantum Rabi model [31-33]. A great effort has been devoted in experiments trying to push the light-matter interaction strength $g$ from the strong coupling regime (with $g$ larger than the dissipation rate) [34-36] to the ultrastrong coupling $(g \sim 0.1 \Omega)[37-40]$ and even the deep strong cou- pling regimes [41]. This model has broad application in cold atoms [42], trapped ions [43-46], quantum dots [47], cavity QED [48, 49] and superconducting circuits $[39,50]$. It plays as a testing ground for strong coupling physics. Moreover, the calculation of its full spectra with the help of integrability is also of general interest [51]. It was shown $[27,29]$ that the phase transition is realized when $\frac{\omega}{\Omega} \rightarrow 0$ at $g_{c}$. In Ref. [29], the critical exponent is shown to be in consistent with the Landau theory. The universal dynamics is also formulated using the KibbleZurek mechanism, which was originally established based on second-order phase transitions $[52,53]$.

The phase transition in the Rabi model can be obtained using the simplest perturbation theory and the effective Hamiltonian approach by some truncation at $T=0[27,29]$. However, it is surprising that while approximations are involved, the predicted critical boundary is shown to be exact. This should not be regarded as some kind of coincidence, which is a long-standing unsolved problem in theory. Here we unveil the underlying origin based on the path-integral approach with Schwinger fermion representation. We demonstrate that the phase transitions in these two models belong to the same universality class by the Landau theory of phase transitions, from which the previous conclusions such as critical exponent and Kibble-Zurek dynamics in consistent with the mean-field theory will become straightforward. We give a unified boundary condition

$$
\omega=\frac{(\eta g)^{2}}{\Omega} \tanh \frac{\beta \Omega}{2}, \quad \frac{\omega}{N \Omega} \rightarrow 0
$$


Physically, it means a classical phase transition since the quantization of the bosonic field is vanishing. The parameter $\eta$ in both models accounts for the effect of rotating wave approximation, in which $\eta=1$ for the presence of it, and $\eta=2$ for its absence. At this point, the fluctuation of the bosonic field is negligible, based on which we derive a much simpler method to study the phase transition in some similar models with interaction between boson fields and atoms, showing that the same phase transition can happen in models with nonidentical atoms, Hubbard interaction and nonlinearity, all of which belong to the Landau paradigm of phase transition. This method is demonstrated to have much broader applicability than the previous approximated approaches.

We implement Eq. 1 using the path-integral approach developed by Popov [54-57], in which the spins are represented by the Schwinger fermions [58, 59]

$$
\sigma_{i}^{+}=\frac{\sigma_{i}^{x}+i \sigma_{i}^{y}}{2}=\alpha_{i}^{\dagger} \beta_{i}, \quad \sigma_{i}^{z}=\alpha_{i}^{\dagger} \alpha_{i}-\beta_{i}^{\dagger} \beta_{i}
$$

Here $\alpha_{i}$ and $\beta_{i}$ are fermion operators, and the number of fermions $\sum_{i} \alpha_{i}^{\dagger} \alpha_{i}+\beta_{i}^{\dagger} \beta_{i}=N_{F}$ is constrained by the number of spins $N_{F}=N$. This is a faithful representation since the Hilbert spaces in these two theories are the same. In the few-spin models, we can even prove that the partition function of the Hamiltonian in these two representations are exactly the same. This is different from the HP method, in which the spin and boson have different Hilbert spaces. The partition function reads as

$$
Z=\operatorname{Tr} \exp (-\beta \mathcal{H})=i^{N} \operatorname{Tr} \exp \left(-\beta \mathcal{H}_{F}-\frac{i \pi}{2} N_{F}\right)
$$

where $N_{F}$ is the constraint defined above. In this new representation, we can take the constraint into account and write the partition function in terms of these fermions as following

$$
Z=i^{N} \int \mathcal{D} \bar{\alpha} \mathcal{D} \alpha \mathcal{D} \bar{\beta} \mathcal{D} \beta \mathcal{D} \bar{b} \mathcal{D} b \mathrm{e}^{-S},
$$

where $S=\int\left(\bar{b} \frac{\partial}{\partial \tau} b+\sum_{i=1}^{N} \bar{\alpha}_{i} \frac{\partial}{\partial \tau} \alpha_{i}+\bar{\beta}_{i} \frac{\partial}{\partial \tau} \beta_{i}+\mathcal{H}\right) d \tau$. We first make a rotating wave approximation to Eq. 1, which corresponds to the Jaynes-Cummings model. Via the fermion coherent representation we have

$$
\mathcal{H}=\omega \bar{b} b+\sum_{i} \frac{\Omega}{2}\left(\bar{\alpha}_{i} \alpha_{i}-\bar{\beta}_{i} \beta_{i}\right)+\frac{g}{\sqrt{N}}\left(\bar{\alpha}_{i} \beta_{i} b+\bar{\beta}_{i} \alpha_{i} \bar{b}\right)+\frac{i \pi}{2 \beta} N_{F}
$$

The trace in Eq. 4 is carried out over different $N_{F}$ spaces of $\mathcal{H}$, in which only the state with $N_{F}=N$ is physical, while all the other modes are canceled exactly $[55,56]$. We solve the above model based on Fourier transformation $b(\tau)=\sum_{n} b_{n} e^{i \omega_{n} \tau}$ and $\psi_{i}(\tau)=\sum_{q} \psi_{i}(q) e^{i \omega_{q} \tau}$, with $\psi_{i}$ for fields $\alpha_{i}$ and $\beta_{i}$, where $\omega_{n}=2 n \pi / \beta, \omega_{q}=(2 q+$ $1) \pi / \beta(n, q \in \mathbb{Z})$ are Matsubara frequencies for bosons and fermions. The total action is decoupled into two parts $S=S_{0}+S_{\text {int }}$, where $S_{0}=\sum_{k, q} \bar{\psi}_{k}(q) G_{0}^{-1}(q) \psi_{k}(q)$, with $\psi_{k}(q)=\left(\alpha_{k}(q), \beta_{k}(q)\right)^{T}$, and

$$
G_{0}(q)=\left(\begin{array}{cc}
\mathcal{G}_{q}^{+} & 0 \\
0 & \mathcal{G}_{q}^{-}
\end{array}\right), \quad \mathcal{G}_{q}^{ \pm}=\frac{1}{\beta\left(i \omega_{q}+i \frac{\pi}{2 \beta} \pm \frac{\Omega}{2}\right)}
$$

The interaction term can be written as $S_{\text {int }}=$ $\sum_{k} \sum_{q, q^{\prime}} \bar{\psi}_{k}(q) \Sigma\left(q-q^{\prime}\right) \psi_{k}\left(q^{\prime}\right)$, where

$$
\Sigma\left(q-q^{\prime}\right)=\frac{g \beta}{\sqrt{N}}\left(\begin{array}{cc}
0 & b_{q-q^{\prime}} \\
\bar{b}_{q^{\prime}-q} & 0
\end{array}\right) .
$$

We see that for the fermion fields, the interacting term is in a quadratic form; while for the bosonic field by treating the fermion field as a Grassmannian constant, the interacting term is just a linear displacement of the bosonic field. We take advantage of this feature and integrate out of the fermion fields $\psi_{i}$, leaving only the bosonic field in the following form $Z=\int \mathcal{D} \bar{b} \mathcal{D} b e^{-S_{\text {eff }}[\bar{b}, b]}$, where

$$
S_{\mathrm{eff}}[\bar{b}, b]=\sum_{n} \beta\left(i \omega_{n}+\omega\right) \bar{b}_{n} b_{n}-N \operatorname{tr} \ln G^{-1},
$$

with $G^{-1}=G_{0}^{-1}+\Sigma$. The previous literature tries to solve the above model from the saddle point solution of $S_{\text {eff }}$ [54-57] and its fluctuation around this point. We choose a different strategy by expanding the solution to infinite orders via Taylor expansion of the bosonic field. In the second term of $S_{\text {eff }}$, we utilize $-\operatorname{tr} \ln G^{-1}=$ $-\operatorname{tr} \ln G_{0}^{-1}+\operatorname{tr} \sum_{m \geq 1} \frac{1}{2 m}\left(G_{0} \Sigma\right)^{2 m}$, which can be represented using the following Feynman diagrams

$$
-\operatorname{tr} \ln G^{-1}=-\operatorname{tr} \ln G_{0}^{-1}+
$$
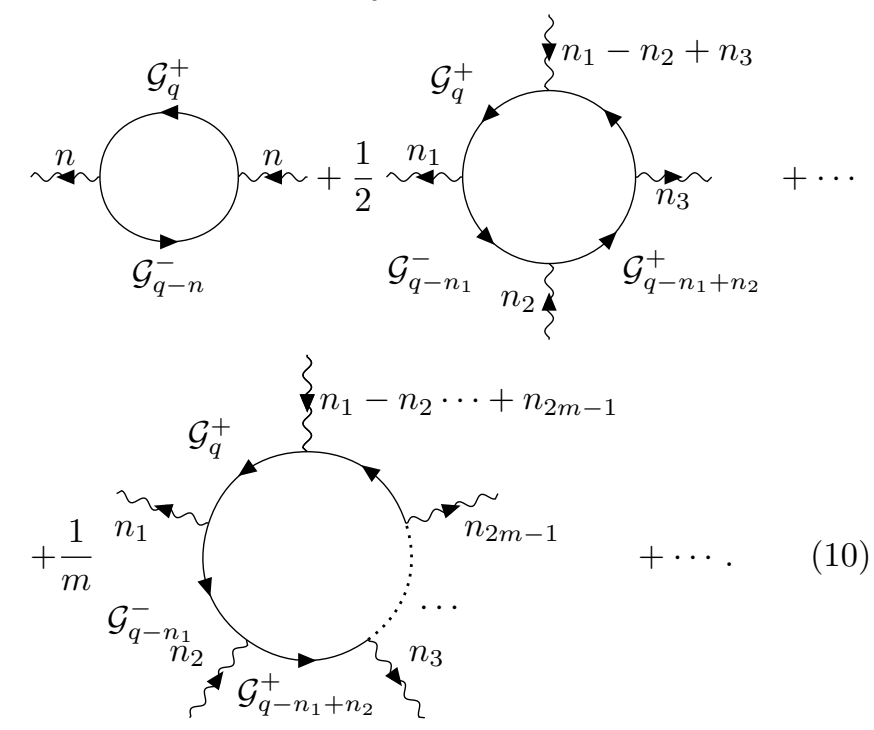

In these diagrams, the bosonic field can be written as

$$
\mathcal{V}_{\left\{n_{i}\right\}}^{(2 m)}=\sum_{\left\{n_{i}\right\}} \chi_{\left\{n_{i}\right\}}^{(2 m)} b_{n_{1}} \bar{b}_{n_{2}} b_{n_{3}} \bar{b}_{n_{4}} \cdots b_{n_{2 m-1}} \bar{b}_{k_{2 m}},
$$

for $k_{2 m}=\sum_{i=1}^{2 m-1}(-1)^{i+1} n_{i}$, in which the Matsubara summation of $\omega_{q}$ is performed. The leading term yields

$$
S_{\mathrm{eff}}^{(2)}=\sum_{n}\left(i \omega_{n}+\omega-\frac{g^{2}}{i \omega_{n}+\Omega} \cdot \tanh \frac{\beta \Omega}{2}\right)\left|b_{n}\right|^{2} .
$$


The real part of this expression, which should be positive for all modes for the normal phase, has been used to determine the superradiant phase transition in the Dicke model [54-57]. It is given by the mode $b_{0}$ by Eq. 2 with $\eta=1$. However, whether the phase transition occurs or not also depends critically on the higher-order terms [60]. The next leading term in $S_{\text {eff }}$ is $\chi_{0}^{(4)}\left|b_{0}\right|^{4}$, where

$$
\chi_{0}^{(4)}=\frac{g \beta}{2 N}\left(\frac{g}{\Omega}\right)^{3}\left(2 \tanh \frac{\beta \Omega}{2}-\beta \Omega \operatorname{sech}^{2} \frac{\beta \Omega}{2}\right) .
$$

We see that $\chi_{0}^{(4)}$ is always a positive number. When $n_{i}$ are different and when all the singular points are first order, we can perform the Matsubara summation of $\omega_{q}$ via the residue theorem and obtain

$$
\chi_{\left\{n_{i}\right\}}^{(4)}=\frac{g^{4} \beta \tanh \frac{\beta \Omega}{2}\left(i \omega_{n_{1}+n_{3}}+2 \Omega\right)}{2 N\left(i \omega_{n_{1}-n_{2}+n_{3}}+\Omega\right) \prod_{i=1}^{3}\left(i \omega_{n_{i}}+\Omega\right)} .
$$

This is a complex value, with its real part to be either positive or negative, depending strongly on the values of $n_{i}$. We find the following upper bound

$$
\left|\chi_{\left\{n_{i}\right\}}^{(4)}\right| \leq \frac{\sqrt{6} g \beta}{N}\left(\frac{g}{\Omega}\right)^{3} \tanh \frac{\beta \Omega}{2} \leq \frac{\sqrt{6} g \beta}{N}\left(\frac{g}{\Omega}\right)^{3} .
$$

This result can be generalized to arbitrary orders. For the $2 m$-th term, we can read from the Feynman diagram as $\chi_{\left\{n_{i}\right\}}^{(2 m)}=\sum_{q} \frac{g^{2 m}}{N^{m-1}} \mathcal{G}_{q}^{+} \mathcal{G}_{q-n_{1}}^{-} \mathcal{G}_{q-n_{1}+n_{2}}^{+} \cdots \mathcal{G}_{k_{2 m}}^{-}$. We noticed that for the higher-order terms, this summation is absolute convergent during the Matsubara summation of $\omega_{q}$. Let us choose a sufficient large $|q|<K_{m} / 2$, then using $\left|\mathcal{G}_{q}^{ \pm}\right| \leq 2 / \beta|\Omega|$, we have

$$
\left|\chi_{\left\{n_{i}\right\}}^{(2 m)}\right|<\frac{K_{m}}{N^{m-1}}\left(\frac{g}{\Omega}\right)^{2 m} .
$$

This upper bound is independent of $n_{i}$. The value of $\chi_{\left\{n_{i}\right\}}^{(2 m)}$ can be calculated using the residue theorem, in which the high order singular points need to be treated carefully. However, this upper bound does not reply on the feature of the singular points, thus has much wider applicability. In general, the large $m$ is, the smaller $K_{m}$ will be for the reason of fast convergence of Matsubara summation of $\omega_{q}$.

This estimation can also be applied to the full Dicke model. In this case, the self-energy $\Sigma$ should be changed accordingly by setting its off-diagonal component $b_{q-q^{\prime}}$ to $\left(b_{q-q^{\prime}}+\bar{b}_{q^{\prime}-q}\right)$ in Eq. 8. However, the propagator $\mathcal{G}_{q}^{ \pm}$is unchanged. Thus the above upper bound is still applicable. We have

$$
S_{\mathrm{eff}}^{(2)}=\sum_{n}\left(i \omega_{n}+\omega\right) \bar{b}_{n} b_{n}-\frac{g^{2} \tanh \frac{\beta \Omega}{2}}{i \omega_{n}+\Omega}\left(b_{n}+\bar{b}_{-n}\right)^{2},
$$

which has the same symmetry - $U(1)$ in the JaynesCummings model with $\eta=1$ and $\mathbb{Z}_{2}$ in the Dicke and
Rabi models with $\eta=2$ - as the original Hamiltonian. The above action yields the boundary in Eq. 2.

This expression can lead to some remarkable results. When $N \rightarrow \infty$, the higher-order terms will approach zero, thus only the leading term $S_{\text {eff }}^{(2)}$ is important. In the few particle case, this limit can be reached via $\frac{g^{2}}{\Omega} \rightarrow 0$, which is equivalent to $\frac{\omega}{\Omega} \rightarrow 0$ using the solution of $g_{c}^{2} \sim \Omega \omega$ at the boundary. This result yields the constraint in Eq. 2 in a unified form, which corresponds to the classical limit of the bosonic field. It has some immediate consequences. In this condition, the higher-order terms of the bosonic field will disappear, leaving only the term $b_{0}$ to be important. Thus we only need to treat the field as a classical variable, for which reason the classical treatment is accurate for the phase transition. Let us assume $b \rightarrow b_{0}$ and $b^{\dagger} \rightarrow b_{0}^{*}$, then

$$
\mathcal{H}=\omega\left|b_{0}\right|^{2}+\frac{\Omega}{2} \sum_{i} \sigma_{i}^{z}+\frac{g}{\sqrt{N}} \sum_{i}^{N}\left(\sigma_{i}^{\dagger} b_{0}+b^{*} \sigma_{i}^{-}\right) .
$$

The $N$ two-level atoms are now independent. We can calculate the free energy of the above model at finite temperature from $Z=e^{-\beta F}=\operatorname{Tr}\left(e^{-\beta \mathcal{H}}\right)$, which yields $F=\omega\left|b_{0}\right|^{2}-\frac{N}{\beta} \ln \frac{\cosh (\beta E)}{2}$ with $E=\sqrt{\Omega^{2} / 4+g^{2}\left|b_{0}\right|^{2} / N}$. Around $b_{0} \sim 0$, we have

$$
F=F_{0}+\left|b_{0}\right|^{2}\left(\omega-\frac{g^{2} \tanh \frac{\beta \Omega}{2}}{\Omega}\right)+\sum_{n \geq 2} F_{2 n}\left|b_{0}\right|^{2 n},
$$

where $F_{0}=-\frac{N}{\beta} \ln \left(2 \cosh \frac{\beta \Omega}{2}\right)$. This result yields Eq. 2 . Here, $b_{0}$ is a classical variable, thus it forbids the superposition of two different states in the spontaneous symmetry breaking phase. The higher-order terms $F_{2 n}$ are ignored in the previous literature for the phase transition [27-30], in which the proof may suffer from loopholes. We can prove that the higher-order terms will scales as $\left(g^{2} /\left(N \Omega^{2}\right)\right)^{n}$, which is in accord with Eq. 16. However, their signs are alternating. When $\beta \Omega \gg 1$, we have

$$
F_{4} \rightarrow \frac{g^{4}}{N \Omega^{3}}, \quad F_{6} \rightarrow-\frac{2 g^{6}}{N^{2} \Omega^{5}}, \quad F_{8} \rightarrow \frac{5 g^{8}}{N^{3} \Omega^{7}} .
$$

In general, $F_{2 n} \sim(-1)^{n} \frac{\Omega}{N^{n-1}}\left(\frac{g}{\Omega}\right)^{2 n}$. The negative coefficients of the higher-order terms may lead to failure of Landau theory of phase transitions (e.g., see the firstorder phase transitions by Landau theory in Ref. 60). To ensure of exact second-order phase transition, one requires that all these coefficients are vanished, which can be achieved only when the constraint in Eq. 2 is satisfied, leaving only the leading term $S_{\text {eff }}^{(2)}$ for instability. In this sense, at the critical point, the fluctuation of the bosonic field is negligible, making our conclusion to be exact. This justifies why even the simplest approximations in the previous literature can yield the accurate phase boundary. It also means that the phase transition 

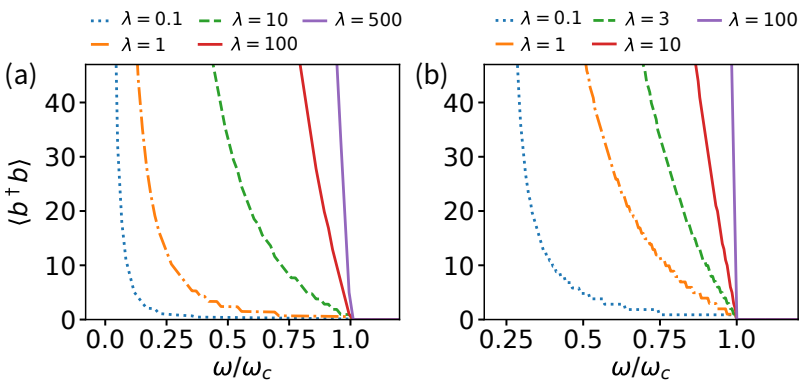

FIG. 1. Phase transitions for model (I) in (a) and model (III) in (b) based on exact diagonalization method at $T=$ 0 . In (a), we choose $N=3,\left(\tilde{g}_{1}, \tilde{g}_{2}, \tilde{g}_{3}\right)=(12,1,100)$, and $\left(\tilde{\Omega}_{1}, \tilde{\Omega}_{2}, \tilde{\Omega}_{3}\right)=(9,50,110)$. Different lines are plotted with $g_{i}=\sqrt{\lambda} \tilde{g}_{i}$ and $\Omega_{i}=\lambda \tilde{\Omega}_{i}$, which yield $\omega_{c}=35.6$ from Eq. 22 . In (b), we choose $N=1, \kappa=0.5, \tilde{g}=12$ and $\tilde{\Omega}=100$, with $\omega_{c}=1.94$ from Eq. 24 using $\lambda$ defined in the same way as (a).

is exactly described by the Landau theory with the number of photon as $\left\langle b^{\dagger} b\right\rangle \sim\left|g-g_{c}\right|^{-\alpha}$, where $\alpha=1 / 2$ is the same as the mean-field theory [27, 29, 61-63].

Our result is useful to understand the phase transitions in the other models with spin and boson interaction for second-order phase transition, in which the HP and the effective Hamiltonian approaches are failed. We discuss several models (I) - (III), which have been justified by exact numerical method with high accuracy (Fig. 1). This approach applies to physics even at finite temperature.

(I) Phase transition in the inhomogeneous model. This model reads as

$$
\mathcal{H}=\omega b^{\dagger} b+\sum_{i} \Omega_{i} \sigma_{i}^{z}+\sum_{i}\left(\frac{g_{i}}{\sqrt{N}} \sigma_{i}^{\dagger} b+\text { h.c. }\right),
$$

for nonidentical atoms interact with a common field. The dynamics in this model has been studied in [64-66]. The phase transition happens at

$$
\omega=\frac{1}{N} \sum_{i=1}^{N} \frac{g_{i}^{2}}{\Omega_{i}} \tanh \frac{\beta \Omega_{i}}{2}, \quad \frac{1}{N} \sum_{i} \frac{g_{i}^{2}}{\Omega_{i}} \rightarrow 0 .
$$

This expression is in consistent with the result in Ref. [67] with inhomogeneous interaction. We confirm this phase transition in Fig. 1 (a), in which a divergent of $\left\langle b^{\dagger} b\right\rangle$ is expected from $S_{\text {eff }}^{(2)}$ across the phase boundary due to the vanished higher-order terms [61].

(II) Anti-rotating term and Hubbard interaction. In this case, we consider the anisotropic interaction of the form of $\left(g_{1} \sigma_{i}^{+}+g_{2} \sigma_{i}\right) b / \sqrt{N}+$ h.c. and Hubbard interaction of $U n(n-1)$, with $n=b^{\dagger} b$. It is frequently termed as anisotropic Rabi model when $g_{1} \neq g_{2}$ [68]. In this case we find the energy level spacing mediated by this term is $\sqrt{\Omega^{2} / 4+\left|g_{1} b_{0}+g_{2} b_{0}^{*}\right|^{2} / N}$, which preserves the $\mathbb{Z}_{2}$ symmetry. The Hubbard term $U$ is unimportant for the phase transition. We have phase transition at

$$
\omega=\frac{\left(g_{1}+g_{2}\right)^{2}}{\Omega} \tanh \frac{\beta \Omega}{2}, \quad \frac{\omega}{N \Omega} \rightarrow 0 .
$$

This condition has been shown in literature [27, 29], and it can be manifested much more straightforward in this work. Thus we have $\eta=2$ in Eq. 2 when all $g_{i}=g$.

(III) Nonlinearity effect. It is inevitable that the higher-order correction by the bosonic field can slightly modify the energy level spacing of the atoms [24]. We mimic this effect using the model $\mathcal{H}=\omega b^{\dagger} b+\sum_{i}(\Omega / 2+$ $\left.\kappa b^{\dagger} b\right) \sigma_{i}^{z}+g / \sqrt{N} \sum_{i}\left(b^{\dagger} \sigma_{i}+\right.$ h.c. $)$, where the term $\kappa$ maybe introduced via the higher-order perturbation theory. This model can not be solved by the HP method for the reason of nonlinear interaction between them. We find the phase transition happens at

$$
\omega=\frac{g^{2}+\kappa N \Omega}{\Omega} \tanh \frac{\beta \Omega}{2}, \quad \frac{\omega}{N \Omega} \rightarrow 0 .
$$

We confirm this phase transition in Fig. 1 (b). This result will have some interesting predictions. When $\kappa$ is independent of $N$, it is relevant, and the phase transition is forbidden in the thermodynamic limit. When $\kappa=\kappa_{0} / N$, which is most likely to happen since the bosonic field is proportional to $1 / \sqrt{N}$, we find that this phase transition is still presented. However, when $\kappa \propto \kappa_{0} / N^{\gamma}$, where $\gamma>1$, this nonlinear effect is irrelevant in the thermodynamic limit, which will not influence the phase boundary. Thus $\gamma=1$ is marginal. This result means that the Dicke phase transition can still happen even taken the nonlinear correction into account.

To conclude, this work is stimulated by the coincident phase boundary in the Dicke and quantum Rabi models, which is exact though derived by some approximated approaches. We explore the underlying origin using the path-integral approah and give a unified boundary condition for these two models, at which the fluctuation of the bosonic field is vanished. In this limit, we can treat the bosonic field as a classical variable, which has much broader applicability than all the above approximated approaches in the determination of phase boundaries in some of the spin and boson interacting models. All these phase transitions belong to the classical Landau theory of phase transition, thus the critical exponent and the associated universal dynamics should be the same as that from the mean-field theory, which can be confirmed using cold atoms, trapped ions and superconducting circuits.

Acknowledgements. This work is supported by the National Natural Science Foundation of China (No. 11774328 and No. 11834005) and the National Key Research and Development Program of China (No. 2016YFA0301700).

* gongm@ustc.edu.cn

[1] R. H. Dicke, "Coherence in Spontaneous Radiation Processes," Phys. Rev. 93, 99-110 (1954).

[2] Michael Tavis and Frederick W. Cummings, "Exact Solu- 
tion for an $N$-Molecule-Radiation-Field Hamiltonian," Phys. Rev. 170, 379-384 (1968).

[3] Klaus Hepp and Elliott H. Lieb, "On the superradiant phase transition for molecules in a quantized radiation field: the Dicke maser model," Annals of Physics 76, 360-404 (1973).

[4] Yo K. Wang and F. T. Hioe, "Phase transition in the Dicke model of superradiance," Phys. Rev. A 7, 831 (1973).

[5] Clive Emary and Tobias Brandes, "Chaos and the quantum phase transition in the Dicke model," Phys. Rev. E 67, 066203 (2003).

[6] Yong Li, Z. D. Wang, and C. P. Sun, "Quantum criticality in a generalized Dicke model," Phys. Rev. A 74, 023815 (2006).

[7] Alexander Altland and Fritz Haake, "Quantum chaos and effective thermalization," Phys. Rev. Lett. 108, 073601 (2012).

[8] Clive Emary and Tobias Brandes, "Quantum Chaos Triggered by Precursors of a Quantum Phase Transition: The Dicke Model," Phys. Rev. Lett. 90, 044101 (2003).

[9] T. Holstein and H. Primakoff, "Field Dependence of the intrinsic Domain Magnetization of a Ferromagnet," Phys. Rev. 58, 1098-1113.

[10] M. J. Bhaseen, J. Mayoh, B. D. Simons, and J. Keeling, "Dynamics of nonequilibrium dicke models," Phys. Rev. A 85, 013817 (2012).

[11] A. L. A L Grimsmo and A. S. Parkins, "Dissipative dicke model with nonlinear atomphoton interaction," J. Phys. B: At. Mol. Opt. Phys. 46, 224012 (2013).

[12] K. Rzażewski, K. Wódkiewicz, and W. Żakowicz, "Phase transitions, two-level atoms, and the $A^{2}$ term," Phys. Rev. Lett. 35, 432 (1975).

[13] Iwo Bialynickibirula and Kazimierz Rzazewski, "No-go theorem concerning the superradiant phase transition in atomic systems," Phys. Rev. A 19, 301-303 (1979).

[14] Pierre Nataf and Cristiano Ciuti, "No-go theorem for superradiant quantum phase transitions in cavity QED and counter-example in circuit QED," Nat. Commun. 1, 72 (2010).

[15] Luca Chirolli, Marco Polini, Vittorio Giovannetti, and Allan H. Macdonald, "Drude weight, cyclotron resonance, and the Dicke model of graphene cavity QED," Phys. Rev. Lett. 109, 267404-267404 (2012).

[16] Kristian Baumann, Christine Guerlin, Ferdinand Brennecke, and Tilman Esslinger, "Dicke quantum phase transition with a superfluid gas in an optical cavity," Nature 464, 1301-1306 (2010).

[17] Kristian Baumann, Rafael Mottl, Ferdinand Brennecke, and Tilman Esslinger, "Exploring symmetry breaking at the Dicke quantum phase transition," Phys. Rev. Lett. 107, 140402 (2011).

[18] R. Mottl, F. Brennecke, K. Baumann, R. Landig, T. Donner, and T. Esslinger, "Roton-type mode softening in a quantum gas with cavity-mediated long-range interactions," Science 336, 1570-1573 (2012).

[19] D. Schmidt, H. Tomczyk, S. Slama, and C. Zimmermann, "Dynamical instability of a Bose-Einstein condensate in an optical ring resonator," Phys. Rev. Lett. 112, 115302 (2014).

[20] Manuele Landini, Nishant Dogra, Katrin Kroeger, Lorenz Hruby, Tobias Donner, and Tilman Esslinger, "Formation of a Spin Texture in a Quantum Gas Cou- pled to a Cavity," Phys. Rev. Lett. 120, 223602-223602 (2018).

[21] Ronen M. Kroeze, Yudan Guo, Varun Vaidya, Jonathan Keeling, and Benjamin Lev, "Spinor self-ordering of a quantum gas in a cavity," Phys. Rev. Lett. 121 (2018).

[22] Markus P. Baden, Kyle J Arnold, Arne L Grimsmo, Scott Parkins, and Murray D. Barrett, "Realization of the Dicke model using cavity-assisted Raman transitions," Phys. Rev. Lett. 113, 020408 (2014).

[23] François Damanet, Andrew J Daley, and Jonathan Keeling, "Atom-only descriptions of the driven-dissipative Dicke model," Phys. Rev. A 99, 033845 (2019).

[24] F. Dimer, B. Estienne, A.S. Parkins, and H.J. Carmichael, "Proposed realization of the dicke-model quantum phase transition in an optical cavity qed system," Phys. Rev. A 75, 013804 (2007).

[25] Chris Hammer, Chunlei Qu, Yongping Zhang, JiaJia Chang, Ming Gong, Chuanwei Zhang, and Peter Engles, "Dicke-type phase transition in a spin-orbitcoupledboseeinstein condensate," Nat. Commun. 5, 4023 (2014).

[26] Pierre Nataf, Thierry Champel, G. Blatter, and D. M. Basko, "Rashba cavity QED: A Route Towards the Superradiant Quantum Phase Transition," Phys. Rev. Lett. 123, 207402 (2019).

[27] Myung-Joong Hwang, Ricardo Puebla, and Martin B. Plenio, "Quantum Phase Transition and Universal Dynamics in the Rabi Model," Phys. Rev. Lett. 115, 180404 (2015).

[28] Ricardo Puebla, Myung-Joong Hwang, Jorge Casanova, and Martin B Plenio, "Probing the dynamics of a superradiant quantum phase transition with a single trapped ion," Phys. Rev. Lett. 118, 073001 (2017).

[29] Maoxin Liu, Stefano Chesi, Zu-Jian Ying, Xiaosong Chen, Hong-Gang Luo, and Hai-Qing Lin, "Universal Scaling and Critical Exponents of the Anisotropic Quantum Rabi Model," Phys. Rev. Lett. 119, 220601 (2017).

[30] S. Ashhab, "Superradiance transition in a system with a single qubit and a single oscillator," Phys. Rev. A 87, 013826 (2013).

[31] Daniel Braak, "Integrability of the Rabi model," Phys. Rev. Lett. 107, 100401 (2011).

[32] Alexander Moroz, "On solvability and integrability of the Rabi model," Ann. Phys. 338, 319-340 (2013).

[33] Murray T. Batchelor and Huan-Qiang Zhou, "Integrability versus exact solvability in the quantum Rabi and Dicke models," Phys. Rev. A 91, 053808 (2015).

[34] A. Wallraff, D. I. Schuster, A. Blais, L. Frunzio, R.-S. Huang, J. Majer, S. Kumar, S. M. Girvin, and R. J. Schoelkopf, "Strong coupling of a single photon to a superconducting qubit using circuit quantum electrodynamics," Nature 431, 162-167 (2004).

[35] M. H. Devoret, S. M. Girvin, and R. J. Schoelkopf, "Circuit-QED: How strong can the coupling between a Josephson junction atom and a transmission line resonator be?" Ann. Phys. 16, 767-779 (2007).

[36] Peter Cristofolini, Gabriel Christmann, Simeon I. Tsintzos, George Deligeorgis, George Konstantinidis, Zacharias Hatzopoulos, Pavlos G. Savvidis, and Jeremy J. Baumberg, "Coupling Quantum Tunneling with Cavity Photons," Science 336, 704-707 (2012).

[37] G. Gnter, A. A. Anappara, J. Hees, A. Sell, G. Biasiol, L. Sorba, S. De Liberato, C. Ciuti, A. Tredicucci, A. Leitenstorfer, and R. Huber, "Sub-cycle switch-on of 
ultrastrong lightmatter interaction," Nature 458, 178181 (2009).

[38] T. Niemczyk, F. Deppe, H. Huebl, E. P. Menzel, F. Hocke, M. J. Schwarz, J. J. Garcia-Ripoll, D. Zueco, T. Hmmer, E. Solano, A. Marx, and R. Gross, "Circuit quantum electrodynamics in the ultrastrong-coupling regime," Nat. Phys. 6, 772-776 (2010).

[39] B. Peropadre, P. Forn-Daz, E. Solano, and J. J. Garca-Ripoll, "Switchable Ultrastrong Coupling in Circuit QED," Phys. Rev. Lett. 105, 023601 (2010).

[40] P. Forn-Daz, J. Lisenfeld, D. Marcos, J. J. Garca-Ripoll, E. Solano, C. J. P. M. Harmans, and J. E. Mooij, "Observation of the Bloch-Siegert Shift in a Qubit-Oscillator System in the Ultrastrong Coupling Regime," Phys. Rev. Lett. 105, 237001 (2010).

[41] J. Casanova, G. Romero, I. Lizuain, J. J. Garca-Ripoll, and E. Solano, "Deep Strong Coupling Regime of the Jaynes-Cummings Model," Phys. Rev. Lett. 105, 263603 (2010).

[42] Helmut Ritsch, Peter Domokos, Ferdinand Brennecke, and Tilman Esslinger, "Cold atoms in cavity-generated dynamical optical potentials," Rev. Mod. Phys. 85, 553 (2013).

[43] Dietrich Leibfried, Rainer Blatt, Christopher Monroe, and David Wineland, "Quantum dynamics of single trapped ions," Reviews of Modern Physics.

[44] D.M. Meekhof, C. Monroe, B.E. King, Wayne M. Itano, and David J. Wineland, "Generation of nonclassical motional states of a trapped atom," Phys. Rev. Lett. 76, 1796 (1996).

[45] H. Häffner, C. F. Roos, and R. Blatt, "Quantum computing with trapped ions," Phys. Rep. 469, 155-203 (2008).

[46] Dingshun Lv, Shuoming An, Zhenyu Liu, Jing-Ning Zhang, Julen S. Pedernales, Lucas Lamata, Enrique Solano, and Kihwan Kim, "Quantum simulation of the quantum rabi model in a trapped ion," Phys. Rev. X 8, 021027 (2018).

[47] Ronald Hanson, Leo P. Kouwenhoven, Jason R. Petta, Seigo Tarucha, and Lieven M.K. Vandersypen, "Spins in few-electron quantum dots," Rev. Mod. Phys. 79, 1217 (2007).

[48] Luigi Garziano, Roberto Stassi, Vincenzo Macri, Anton Frisk Kockum, Salvatore Savasta, and Franco Nori, "Multiphoton quantum Rabi oscillations in ultrastrong cavity QED," Phys. Rev. A 92, 063830 (2015).

[49] C. K. Law and J. H. Eberly, "Arbitrary Control of a Quantum Electromagnetic Field," Phys. Rev. Lett. 76, 1055-1058 (1996).

[50] Oliver Viehmann, Jan von Delft, and Florian Marquardt, "Superradiant phase transitions and the standard description of circuit QED," Phys. Rev. Lett. 107, 113602 (2011).

[51] Qiongtao Xie, Honghua Zhong, Murray T. Batchelor, and Chaohong Lee, "The quantum rabi model: solution and dynamics," J. Phys. A: Math. Theor. 50, 113001 (2017).

[52] W. H. Zurek, "Cosmological experiments in superfluid helium?" Nature 317, 505 - 508 (1985).

[53] W. H. Zurek, "Cosmological experiments in condensed matter physics," Physics Reports 276, 177 - 221 (1996).

[54] V. N. Popov and S. A. Fedotov, "Behavior of the partition function of Dicke type models in the limit of a large number of atoms," Theor. Math. Phys. 51, 363371 (1982).
[55] M. Aparicio Alcalde and B. M. Pimentel, "Path integral approach to the full Dicke model," Physica A 390, 33853396 (2011).

[56] Victor Nikolaevich Popov and S. A. Fedotov, "The functional integration method and diagram technique for spin systems," Sov. Phys. JETP 67, 535-541 (1988).

[57] Viktor N. Popov and Victor Nikolaevich Popov, Functional integrals and collective excitations (Cambridge University Press, 1991).

[58] Ganapathy Baskaran, Z Zou, and Philip W Anderson, "The resonating valence bond state and high-tc superconductivitya mean field theory," Solid state communications 63, 973-976 (1987).

[59] Xiao-Gang Wen and Patrick A. Lee, "Theory of underdoped cuprates," Phys. Rev. Lett. 76, 503 (1996).

[60] First order phase transition can be realized by Landau theory in the following model $f(T)=f_{0}+\alpha\left(T-T_{c}\right) m^{2}+$ $\beta m^{4}+\gamma m^{4}$, where $\beta<0$ and $\alpha>0, \gamma>0$.

[61] Peter Kirton, Mor M. Roses, Jonathan Keeling, and Emanuele G. Dalla Torre, "Introduction to the Dicke model: From equilibrium to nonequilibrium, and vice versa," Adv. Quantum Technol. 2, 1800043 (2019).

[62] D. L. Nagy, G. Szirmai, and P. Domokos, "Critical exponent of a quantum-noise-driven phase transition: The open-system Dicke model," Phys. Rev. A 84, 043637 (2011).

[63] Dávid Nagy and Péter Domokos, "Critical exponent of quantum phase transitions driven by colored noise," Phys. Rev. A 94, 063862 (2016).

[64] Oleksandr Tsyplyatyev and Daniel Loss, "Dynamics of the inhomogeneous Dicke model for a single-boson mode coupled to a bath of nonidentical spin- $1 / 2$ systems," Phys. Rev. A 80, 023803 (2009).

[65] Christoph Sträter, Oleksandr Tsyplyatyev, and Alexandre Faribault, "Nonequilibrum dynamics in the strongly excited inhomogeneous Dicke model," Phys. Rev. B 86, 195101 (2012).

[66] L.J. Zou, David Marcos, Sebastian Diehl, Stefan Putz, Jörg Schmiedmayer, Johannes Majer, and Peter Rabl, "Implementation of the Dicke lattice model in hybrid quantum system arrays," Phys. Rev. Lett. 113, 023603 (2014).

[67] F. T. Hioe, "Phase transitions in some generalized Dicke models of superradiance," Phys. Rev. A 8, 1440 (1973).

[68] Qiong-Tao Xie, Shuai Cui, Jun-Peng Cao, Luigi Amico, and Heng Fan, "Anisotropic Rabi model," Phys. Rev. X 4, 021046 (2014). 